\title{
Dimensions of the Julia sets of rational maps with the backward contraction property
}

\author{
by
}

Huaibin Li and Weixiao Shen (Hefei)

\begin{abstract}
Consider a rational map $f$ on the Riemann sphere of degree at least 2 which has no parabolic periodic points. Assuming that $f$ has Rivera-Letelier's backward contraction property with an arbitrarily large constant, we show that the upper box dimension of the Julia set $J(f)$ is equal to its hyperbolic dimension, by investigating the properties of conformal measures on the Julia set.
\end{abstract}

1. Introduction. Let $f: \overline{\mathbb{C}} \rightarrow \overline{\mathbb{C}}$ be a rational map of degree $d \geq 2$ on the Riemann sphere. We are interested in the fractal properties of the Julia set $J(f)$. It is well known that in the case that $f$ is hyperbolic, all possible dimensions coincide. In [4], this result was generalized to all rational maps which satisfy a summability condition. See [11] for more historical remarks and advances in this direction.

The summability condition and the stronger Collet-Eckmann condition can be considered as non-uniform hyperbolicity conditions. As shown by J. Rivera-Letelier [9], they imply a backward contraction condition (see the definition below) which was first introduced therein.

In the following, all the distances, diameters and norms of derivatives are measured using the spherical metric and $B(z, r)$ denotes a ball of radius $r$ centered at $z$. Let $\operatorname{Crit}(f)$ denote the set of critical points of $f$ and let

$$
\operatorname{Crit}^{\prime}(f)=\operatorname{Crit}(f) \cap J(f) .
$$

For every $c \in \operatorname{Crit}(f)$ and $\delta>0$ we denote by $\widetilde{B}(c, \delta)$ the connected component of $f^{-1}(B(f(c), \delta))$ that contains $c$.

2000 Mathematics Subject Classification: Primary 37F35.

Key words and phrases: Julia set, conformal measure, rational map, hyperbolic dimension, box dimension, backward contracting map.

WS is supported by the "Bai Ren Ji Hua" project of the CAS and the 973 project 2006CB805900. We would like to thank the referee for carefully reading the manuscript. 
Definition 1. Given a constant $r>1$, we say that $f$ has the backward contraction property with constant $r(f \in \mathrm{BC}(r)$ for short $)$ if there exists $\delta_{0}>0$ such that for every $c \in \operatorname{Crit}^{\prime}(f)$, every $0<\delta \leq \delta_{0}$, every integer $n \geq 1$ and every component $W$ of $f^{-n}(\widetilde{B}(c, r \delta))$, we have

$$
\operatorname{dist}(W, \mathrm{CV}(f)) \leq \delta \Rightarrow \operatorname{diam}(W)<\delta,
$$

where $\mathrm{CV}(f)=f(\operatorname{Crit}(f))$. If $f \in \mathrm{BC}(r)$ for every $r>1$, we will say that $f \in \mathrm{BC}(\infty)$.

We call a compact forward invariant subset $X$ of $\overline{\mathbb{C}}$ hyperbolic if there exist $C>0$ and $\lambda>1$ such that for every $n \geq 1$ and every $z \in X$,

$$
\left|D f^{n}(z)\right| \geq C \lambda^{n}
$$

Clearly, a hyperbolic set is contained in the Julia set.

For a compact set $X \subset \overline{\mathbb{C}}$, let $\operatorname{HD}(X)$ denote its Hausdorff dimension. The hyperbolic dimension $\operatorname{HD}_{\text {hyp }}(f)$ of $f$ is the supremum of the Hausdorff dimensions of hyperbolic subsets of $J(f)$, i.e.

$$
\operatorname{HD}_{\text {hyp }}(f)=\sup \{\operatorname{HD}(X): X \text { is a hyperbolic subset of } J(f)\} \text {. }
$$

Clearly, $\operatorname{HD}_{\text {hyp }}(f) \leq \mathrm{HD}(J(f))$.

The main goal of this paper is to prove the following theorem.

MaIn Theorem. Let $f: \overline{\mathbb{C}} \rightarrow \overline{\mathbb{C}}$ be a rational map of degree at least 2 without parabolic periodic points. If $f \in \mathrm{BC}(\infty)$, then the upper box dimension $\overline{\mathrm{BD}}(J(f))$ of the Julia set is equal to the hyperbolic dimension of $f$ :

$$
\overline{\mathrm{BD}}(J(f))=\mathrm{HD}(J(f))=\operatorname{HD}_{\text {hyp }}(f) .
$$

For the definition of the upper and lower box dimensions and the Hausdorff dimension, see [3]. Let us mention the following well-known inequality: $\mathrm{HD}(X) \leq \underline{\mathrm{BD}}(X) \leq \overline{\mathrm{BD}}(X)$.

The proof of the Main Theorem is based on analyzing the regularity of conformal measures. Recall that a probability measure $\mu$ on $J(f)$ is said to be $t$-conformal for $f$ if for every Borel set $A \subset J(f)$ such that $\left.f\right|_{A}$ is injective, we have

$$
\mu(f(A))=\int_{A}\left|f^{\prime}\right|^{t} d \mu .
$$

The number $t$ is called the exponent of the conformal measure. The minimum exponent, denoted by $\delta_{*}(f)$, is the infimum of the exponents of conformal measures on the Julia set $J(f)$ :

$$
\delta_{*}(f)=\inf \{t \text { : there is a } t \text {-conformal measure on } J(f)\} .
$$

Conformal measures were introduced in holomorphic dynamics by Sullivan [10], who proved the existence of at least one such measure on $J(f)$. Denker, Urbański and Przytycki (see $[2,8]$ ) proved that for any rational map 
$f$ of degree at least 2 , the hyperbolic dimension is equal to the minimum exponent, i.e.

$$
\delta_{*}(f)=\operatorname{HD}_{\text {hyp }}(f) \leq \mathrm{HD}(J(f)) .
$$

The crucial step in obtaining the Main Theorem is to prove the following theorem.

THEOREM 1. Let $f$ be a rational map of degree $d \geq 2$ which satisfies $\mathrm{BC}(\infty)$. Assume that

(*) any forward invariant compact subset of $J(f)$ containing no critical points is hyperbolic.

Let $\mu$ be a t-conformal measure on $J(f)$. Then for any $\alpha>0$, there exists $\varepsilon_{0}>0$ such that for any $\varepsilon \leq \varepsilon_{0}$ and any $z \in J(f)$, we have

$$
\frac{\mu(B(z, \varepsilon))}{\varepsilon^{t}} \geq \varepsilon^{\alpha}
$$

It is not clear if the condition $(*)$ holds for all rational maps without parabolic periodic points and satisfying the $\mathrm{BC}(\infty)$ condition. Nevertheless, as Proposition 8.1 in $[9]$ shows, it holds if $J(f) \neq \overline{\mathbb{C}}$. In the remaining case, the main theorem reduces to the statement that $\operatorname{HD}_{\text {hyp }}(f)=2$.

Remark 1. Assume furthermore that $J(f) \neq \overline{\mathbb{C}}$. Then by Theorem B of [9], $J(f)$ has zero area. By Corollary 8.3 of [9], $f$ has neither Siegel disks nor Hermann rings. So by Fact 8.1 and Lemma 8.2 of $[4], \overline{\mathrm{BD}}(J(f))=\delta_{\mathrm{cr}}(f)$, where $\delta_{\mathrm{cr}}(f)$ is the Poincaré exponent. Therefore, in this case, we obtain the following equalities:

$$
\overline{\mathrm{BD}}(J(f))=\underline{\mathrm{BD}}(J(f))=\operatorname{HD}(J(f))=\operatorname{HD}_{\text {hyp }}(f)=\delta_{*}(f)=\delta_{\mathrm{cr}}(f) .
$$

\section{Background}

2.1. Koebe distortion. We shall use the following version of the Koebe distortion theorem that appeared in [7].

Koebe Principle. There exists $r(f)>0$, depending on $f$, and for each $\varepsilon \in(0,1)$ there exists a constant $K(\varepsilon)>1$ such that the following property holds. Let $x \in J(f), n \geq 0$ and $r \in(0, r(f))$. Suppose that $f^{n}: W \rightarrow$ $B(x, r)$ is a conformal map. Then for every $z_{1}, z_{2} \in W$ with $f^{n}\left(z_{1}\right), f^{n}\left(z_{2}\right) \in$ $B(x, \varepsilon r)$, we have

$$
\frac{\left|\left(f^{n}\right)^{\prime}\left(z_{1}\right)\right|}{\left|\left(f^{n}\right)^{\prime}\left(z_{2}\right)\right|} \leq K(\varepsilon)
$$

Moreover, $K(\varepsilon) \rightarrow 1$ as $\varepsilon \rightarrow 0$.

2.2. Backward contracting rational maps. We collect a few results about rational maps satisfying the backward contraction property. These results were proved in [9]. 
Lemma 1 ([9, Theorem B]). Let $f$ be a rational map of degree at least 2 . Then there is a constant $r>1$, only depending on the degree of $f$, such that if $f$ satisfies $\mathrm{BC}(r)$, then the following properties hold:

1. If $J(f) \neq \overline{\mathbb{C}}$, then $J(f)$ has zero Lebesgue measure.

2. If $J(f)=\overline{\mathbb{C}}$, then there is a set of full Lebesgue measure of points in $\overline{\mathbb{C}}$ whose forward orbit accumulates on a critical point of $f$.

An open set $V$ is called nice if $f^{n}(\partial V) \cap V=\emptyset$ for all $n \geq 0$. A puzzle neighborhood $V$ of $\mathrm{Crit}^{\prime}(f)$ is a nice open set $V=\bigcup_{c \in \mathrm{Crit}^{\prime}(f)} V_{c}$, where $V_{c}$ 's are pairwise disjoint Jordan disks.

Lemma 2 ([9, Lemma 6.2]). Let $f: \overline{\mathbb{C}} \rightarrow \overline{\mathbb{C}}$ be a rational map of degree two or more such that $f \in \mathrm{BC}(\infty)$. Then there exists $\varepsilon_{0}>0$ such that for every $\varepsilon \in\left(0, \varepsilon_{0}\right]$, there exists a puzzle neighborhood $V=\bigcup_{c \in \operatorname{Crit}^{\prime}(f)} V_{c}$ of $\operatorname{Crit}^{\prime}(f)$ with

$$
\widetilde{B}(c, \varepsilon) \subset V_{c} \subset \widetilde{B}(c, 2 \varepsilon) .
$$

Lemma 3 ([9, Proposition 8.1]). Let $f$ be a rational map of degree two or more such that $f \in \mathrm{BC}(\infty)$ and the set

$$
\left\{z \in \overline{\mathbb{C}}: \omega(z) \cap \operatorname{Crit}^{\prime}(f)=\emptyset\right\}
$$

has positive Lebesgue measure. If $K \subset J(f)$ is a compact and forward invariant set which contains neither critical points nor parabolic periodic points, then $K$ is a hyperbolic set.

3. Some preparation. In what follows, let $f: \overline{\mathbb{C}} \rightarrow \overline{\mathbb{C}}$ be a rational map of degree $d \geq 2$ without parabolic points such that $f \in \operatorname{BC}(\infty)$. Let $\ell_{\max }$ be the maximum of the orders of the critical points.

Given a nice set $V$, we will say a connected set $U$ is a critical pull back of $V$ if there exists $n \geq 1$ such that $U$ is a connected component of $f^{-n}(V)$ and $U \cap \operatorname{Crit}(f) \neq \emptyset$.

For a nice set $V$, we define

$$
D(V)=\left\{z \in \overline{\mathbb{C}}: \exists k \geq 0 \text { such that } f^{k}(z) \in V\right\} .
$$

Each connected component of $D(V)$ is called a landing domain of $V$; for any $z \in D(V)$, the smallest integer $k \geq 0$ with $f^{k}(z) \in V$ is called the landing time of $z$ into $V$.

Proposition 2. For any $\beta \in\left(0,1 / \ell_{\max }\right)$, there exists $C(\beta)>0$ such that for every $c \in \operatorname{Crit}^{\prime}(f), n \in \mathbb{N}$ and $\varepsilon$ sufficiently small, if $W$ is a component of $f^{-n}(\widetilde{B}(c, \varepsilon))$, then

$$
\operatorname{diam}(W) \leq C(\beta) \varepsilon^{\beta} .
$$


Proof. Fix a large number $r>1$. By Lemma 2, for each $k \geq 0$, there exists a puzzle neighborhood $\widetilde{V}_{k}$ of $\operatorname{Crit}^{\prime}(f)$ such that

$$
\widetilde{B}\left(c, \varepsilon_{0} / r^{k}\right) \subset \widetilde{V}_{k}(c) \subset \widetilde{B}\left(c, 2 \varepsilon_{0} / r^{k}\right),
$$

where $\varepsilon_{0}>0$ is a small number. By choosing $\varepsilon_{0}$ smaller if necessary, we may assume that any critical pull back of $\widetilde{V}_{k-1}$ is contained in $\widetilde{V}_{k}$, since $f$ satisfies $\mathrm{BC}(2 r)$. Moreover, we can find a periodic orbit $X$ with at least two points outside $V_{0}$. Clearly, $D\left(V_{0}\right) \cap X=\emptyset$.

It suffices to prove that for any $\beta \in\left(0,1 / \ell_{\max }\right)$ there exists $C>0$ such that for any landing domain $U$ of some $\widetilde{V}_{n}$, we have

$$
\operatorname{diam}(U) \leq C r^{-n \beta} \text {. }
$$

Fix $z \in D\left(\widetilde{V}_{n}\right)$. For each $k=0,1, \ldots, n$, let $s_{k}$ be the landing time of $z$ into $\widetilde{V}_{k}$ and let $U_{k}$ be the landing domain of $\widetilde{V}_{k}$ which contains $z$. Then $U_{k} \subset U_{k-1}$. Let $U_{k-1}^{\prime}$ be the component of $\left(f^{s_{k}}\right)^{-1}\left(\widetilde{V}_{k-1}\right)$ containing $z$. Then

$$
U_{k} \subset U_{k-1}^{\prime} \subset U_{k-1} \text {. }
$$

Claim. $f^{s_{k}}: U_{k-1}^{\prime} \rightarrow \widetilde{V}_{k-1}$ is conformal.

Indeed, otherwise there exists $0 \leq s<s_{k}$ such that $W=f^{s}\left(U_{k-1}^{\prime}\right)$ contains a critical point $c^{\prime}$. But as we noted above, this would imply that $W \subset \widetilde{V}_{k}$, which contradicts the fact that $s_{k}$ is the landing time of $z$ into $\widetilde{V}_{k}$.

Thus,

$$
\bmod \left(U_{k-1} \backslash U_{k}\right) \geq \bmod \left(U_{k-1}^{\prime} \backslash U_{k}\right) \geq \inf _{c \in \mathrm{Crit}^{\prime}(f)} \bmod \left(\widetilde{V}_{k-1}(c) \backslash \widetilde{V}_{k}(c)\right) .
$$

For any $r \geq 4$, there exists $L(r)>1$ such that for every $c \in \operatorname{Crit}^{\prime}(f)$, we have

$$
\bmod \left(\widetilde{V}_{k-1}(c) \backslash \widetilde{V}_{k}(c)\right) \geq \frac{1}{L(r) \ell_{\max }} \log r
$$

Moreover, $L(r) \rightarrow 1$ as $r \rightarrow \infty$.

Hence, by the Grötzsch inequality (see for example [5, Corollary B.5]) we have

$$
\begin{aligned}
\bmod \left(U_{0} \backslash U_{n}\right) & \geq \sum_{k=1}^{n} \bmod \left(U_{k-1} \backslash U_{k}\right) \geq \sum_{k=1}^{n} \inf _{c} \bmod \left(\widetilde{V}_{k-1}(c) \backslash \widetilde{V}_{k}(c)\right) \\
& \geq \frac{1}{L(r) \ell_{\max }} n \log r .
\end{aligned}
$$

Since $U_{0} \cap X=\emptyset$, the diameter of $\overline{\mathbb{C}} \backslash U_{0}$ is bounded away from zero. It follows that $\operatorname{diam}\left(U_{n}\right) \leq C r^{-n / L(r) \ell_{\max }}$, where $C$ is a constant. The proof is complete. 
Lemma 4. If the set $\left\{z \in \overline{\mathbb{C}}: \omega(z) \cap \operatorname{Crit}^{\prime}(f)=\emptyset\right\}$ has positive Lebesgue measure, then for any $\delta>0$ there exists $\eta>0$ such that if $W$ is a connected set intersecting the Julia set, and $\operatorname{diam}\left(f^{n}(W)\right)<\eta$ for some $n \geq 0$, then

$$
\operatorname{diam}(W)<\delta .
$$

Proof. By Proposition 2, there exists a neighborhood $V_{0}$ of $\mathrm{Crit}^{\prime}(f)$ such that any pull back of $V_{0}$ has diameter smaller than any given number $\delta>0$. Let $V \Subset V_{0}$ be another neighborhood of $\operatorname{Crit}^{\prime}(f)$.

Define

$$
K(V)=\left\{z \in J(f): f^{m}(z) \notin V, m=0,1,2, \ldots\right\} .
$$

By Lemma 3, $K(V)$ is a hyperbolic set of $f$. So there exists $m_{0}$ such that for any $z \in K(V)$ we have

$$
\left|\left(f^{m_{0}}\right)^{\prime}(z)\right|>2 .
$$

In particular, for any $z \in K(V), f^{m_{0}}$ is univalent in a neighborhood of $z$. By continuity, there exists $\eta_{0} \in(0, \operatorname{diam}(\overline{\mathbb{C}}))$ such that for each $z_{0} \in K(V)$, $f^{m_{0}} \mid B\left(z_{0}, 3 \eta_{0}\right)$ is univalent and the above inequality holds for all $z \in$ $B\left(z_{0}, 3 \eta_{0}\right)$. Let

$$
U=\left\{z \in \overline{\mathbb{C}}: d(z, K(V))<\eta_{0} / 2\right\}
$$

Then if $A$ is a connected subset of $\overline{\mathbb{C}}$ which intersects $U$, then

$$
\operatorname{diam}\left(f^{m_{0}}(A)\right) \geq \min \left(2 \operatorname{diam}(A), \eta_{0}\right) .
$$

To see this, take $z_{0} \in U$ with $B\left(z_{0}, \eta_{0} / 2\right) \cap A \neq \emptyset$. If $A \subset B\left(z_{0}, \eta_{0}\right)$, then $\operatorname{diam}\left(f^{m_{0}}(A)\right) \geq 2 \operatorname{diam}(A)$, and otherwise $\operatorname{diam}\left(f^{m_{0}}(A)\right) \geq \eta_{0}$.

Claim. There exists $N$ such that for every $z \in J(f) \backslash U$, there exists $n(z) \leq N$ such that $f^{n(z)}(z) \in V$.

Indeed, $\left\{f^{-j}(V)\right\}_{j=0}^{\infty}$ is an open covering of the compact set $J(f) \backslash U$, so there exists $N$ such that

$$
\bigcup_{j=0}^{N} f^{-j}(V) \supset J(f) \backslash U .
$$

The claim is proved.

Now let $z \in J(f)$ and $W \ni z$ be a connected set with $\operatorname{diam}\left(f^{n}(W)\right)<\eta_{0}$.

CASE 1: $f^{k}(W) \subset U$ for all $k=0,1, \ldots, n-1$. Write $n=q m_{0}+r$, $0 \leq r<m_{0}$. By $(2)$, we obtain

$$
\operatorname{diam}\left(f^{r}(W)\right) \leq \operatorname{diam}\left(f^{n}(W)\right) / 2^{q} .
$$

It follows that $\operatorname{diam}(W)<\delta$ provided that $\operatorname{diam}\left(f^{n}(W)\right)$ is small enough.

CASE 2: There exists a largest $k \leq n-1$ such that $f^{k}(W) \not \subset U$. As in Case 1, $\operatorname{diam}\left(f^{k+1}(W)\right)$ is small, hence $\operatorname{diam}\left(f^{k}(W)\right)$ is small. By the claim above, there exists $s \leq N$ such that $f^{k+s}(W) \cap V \neq \emptyset$. Provided that 
$\operatorname{diam}\left(f^{n}(W)\right)$ is small enough, $\operatorname{diam}\left(f^{k+s}(W)\right)<d\left(\partial V, \partial V_{0}\right)$, which implies that $f^{k+s}(W) \subset V_{0}$, hence $\operatorname{diam}(W)<\delta$.

Given an open set $\Omega \subset \mathbb{C}$ and $z \in \Omega$, let

$$
\operatorname{IR}(\Omega, z)=\inf _{w \in \partial \Omega} d(z, w) \text { and } \operatorname{OR}(\Omega, z)=\sup _{w \in \partial \Omega} d(z, w) .
$$

Proposition 3. Let $f$ be a rational map of degree $d \geq 2$. For any $\eta \in$ $(0, \operatorname{diam}(\mathbb{C}) / 2)$ and $\varepsilon \in(0, \eta)$ and for any $z \in J(f)$, there exist $n_{0} \in \mathbb{N} \cup\{0\}$ and $\eta^{\prime}$ such that:

- $C \eta \leq \eta^{\prime} \leq \eta$, where $C=C(f)$ is a constant;

- letting $W_{n_{0}}$ be the pull-back of $B\left(f^{n_{0}}(z), \eta^{\prime}\right)$ under $f^{n_{0}}$ to $z$, we have

$$
\operatorname{IR}\left(W_{n_{0}}, z\right)=\varepsilon
$$

Proof. We consider the pull-back $\widehat{W}_{n}$ of the disk $B\left(f^{n}(z), \eta\right)$ along $\operatorname{orb}(z)$ to $z$. Then

$$
\operatorname{IR}\left(\widehat{W}_{n}, z\right) \rightarrow 0 \quad \text { as } n \rightarrow \infty,
$$

for otherwise there would be a ball $B$ centered at $z$ such that $\operatorname{diam}\left(f^{n}(B)\right)$ $\leq 2 \eta$, which would imply that $z \in B \subset \mathbb{C} \backslash J(f)$.

Thus there exists a positive $n_{0} \in \mathbb{N}$ such that

$$
\operatorname{IR}\left(\widehat{W}_{n_{0}}, z\right) \geq \varepsilon \quad \text { but } \operatorname{IR}\left(\widehat{W}_{n_{0}+1}, z\right)<\varepsilon
$$

Now let $W^{\prime}$ be the component of $f^{-1}\left(B\left(f^{n_{0}+1}(z), \eta\right)\right)$ containing $f^{n_{0}}(z)$. Then $W^{\prime} \not \supset B\left(f^{n_{0}}(z), \eta\right)$. It follows that $\bar{\eta}:=\operatorname{IR}\left(W^{\prime}, f^{n_{0}}(z)\right) \leq \eta$. Clearly, $\bar{\eta} \geq C \eta$, where $C=\left(\max \left|f^{\prime}\right|\right)^{-1}$.

Let $\Omega(t)$ be the component of $f^{-n_{0}}\left(B\left(f^{n_{0}}(z), t\right)\right)$ containing $z$ and consider the map $h(t)=\operatorname{IR}(\Omega(t), z)$. Since $h(t)$ is continuous and $h(\eta) \geq \varepsilon$, $h(\bar{\eta})<\varepsilon$, there exists $\eta^{\prime} \in(\bar{\eta}, \eta]$ such that $h\left(\eta^{\prime}\right)=\varepsilon$. This completes the proof.

Proposition 4. Let $f$ be a rational map. There exists $C>0$ such that for every $z \in \overline{\mathbb{C}}$ and every small neighborhood $U$ of $z$,

$$
\frac{\mathrm{OR}(U, z)}{\operatorname{IR}(U, z)} \leq C \frac{\mathrm{OR}(f(U), f(z))}{\operatorname{IR}(f(U), f(z))} .
$$

Proof. By the Koebe principle, it suffices to consider $U$ contained in a small neighborhood of a critical point of $f$. Since near a critical point, $f$ behaves like a polynomial $z \mapsto z^{k}$, the proposition follows easily.

4. Proof of Theorem 1. In this section, we prove Theorem 1. We shall use the following notion introduced in [1].

Definition 2. A sequence $\left\{G_{k}\right\}_{k=0}^{n}$ of connected open sets is called a quasi-chain if $f\left(G_{k}\right) \supset G_{k+1}$ for each $0 \leq k<n$. The order of the quasi-chain is the number of $k \in\{0,1, \ldots, n-1\}$ such that $G_{k}$ contains a critical point. 
We shall also need the following lemma related to Lemma 1.3 of [6].

Lemma 5. Let $f: \overline{\mathbb{C}} \rightarrow \overline{\mathbb{C}}$ be a rational map of the Riemann sphere of degree at least two and let $\mu$ be a t-conformal measure on $J(f)$. Then there exists a constant $C>0$ (depending on $f$ ) such that if $V$ is a connected open set and $U$ is a component of $f^{-1}(V)$, then

$$
\frac{\mu(U)}{\operatorname{diam}(U)^{t}} \geq C \frac{\mu(V)}{\operatorname{diam}(V)^{t}} .
$$

Proof. By Lemma 1.3 in [6], we have

$$
\frac{\operatorname{diam}(V)}{\operatorname{diam}(U)} \geq C \sup \left\{\left|f^{\prime}(z)\right|: z \in U\right\} .
$$

By the $t$-covariance of $\mu$, the lemma follows easily.

In the following,

$$
\widetilde{B}(\delta)=\bigcup_{c \in \operatorname{Crit}^{\prime}(f)} \widetilde{B}(c, \delta) .
$$

Proof of Theorem 1. Fix $\alpha \in(0,1)$. Let $r>4^{\ell_{\max }}$ be a constant to be determined. Since $f$ satisfies $\mathrm{BC}(r)$, there exists $\delta_{0}$ such that for any $\delta \in\left(0, \delta_{0}\right)$, if $U$ is a critical pull back of $\widetilde{B}(r \delta)$ then $U \subset \widetilde{B}(\delta)$.

By Lemma 4, there exists $\eta>0$ such that any pull back of a ball of radius $2 \eta$ has diameter less than $\delta_{0}$. By Proposition 3, for any $\varepsilon<\eta$ there exist $\eta^{\prime} \asymp \eta\left(\eta^{\prime} \leq \eta\right)$ and $n$ such that the component $W$ of $f^{-n}\left(B\left(f^{n}(z), \eta^{\prime}\right)\right)$ which contains $z$ satisfies

$$
\operatorname{IR}(W, z)=\varepsilon^{1+\alpha / 2 t}
$$

Let $W_{k}=f^{k}(W), k=0,1, \ldots, n$.

We shall prove that there exist constants $C \in(0,1)$ and $\theta>0$ depending only on $f$ such that

$$
\begin{aligned}
\frac{\mu(W)}{\operatorname{diam}(W)^{t}} & \geq C \varepsilon^{\theta \gamma}, \\
\frac{\mathrm{OR}(W, z)}{\operatorname{IR}(W, z)} & \leq\left(C \varepsilon^{\theta \gamma}\right)^{-1},
\end{aligned}
$$

where $\gamma=2 \ell_{\max } /\left(\log r-\ell_{\max } \log 4\right)$.

Choosing $r$ large enough, we have $\theta \gamma \leq \alpha / 3$. Provided that $\varepsilon>0$ is small enough, we have $C \varepsilon^{\theta \gamma} \geq \varepsilon^{\alpha / 2}$. Thus (4) implies $\operatorname{OR}(W, z) \leq \varepsilon$, so $\mu(B(z, \varepsilon)) \geq \mu(W)$; together with inequality (3) we have

$$
\mu(B(z, \varepsilon)) \geq \mu(W) \geq \varepsilon^{t+\alpha},
$$

as we wished.

If $f^{n} \mid W$ extends to a conformal map onto $B\left(f^{n}(z), 2 \eta\right)$ then the inequalities follow easily from the Koebe principle and the $t$-covariance of $\mu$. 
In order to deal with the general case, we define a quasi-chain $\left\{\widehat{W}_{k}\right\}_{k=0}^{n}$ by the following rules: (i) $\widehat{W}_{n}=B\left(f^{n}(z), 2 \eta\right)$; (ii) once $\widehat{W}_{k+1} \ni f^{k+1}(z)$ is defined, let $\widehat{W}_{k}^{\prime}$ be the connected component of $f^{-1}\left(\widehat{W}_{k+1}\right)$ which contains $f^{k}(z)$; (iii) if $\widehat{W}_{k}^{\prime}$ contains no critical point, then $\widehat{W}_{k}=\widehat{W}_{k}^{\prime}$, otherwise

$$
\widehat{W}_{k}=B\left(f^{k}(z), 2 \operatorname{diam}\left(\widehat{W}_{k}^{\prime}\right)\right) .
$$

Let $m$ be the order the quasi-chain $\left\{\widehat{W}_{k}\right\}_{k=0}^{n}$. Let $n_{0}=n$ and let $n_{1}>$ $\cdots>n_{m}$ be all integers in $\{0,1, \ldots, n-1\}$ such that $\widehat{W}_{n_{i}}$ contains a critical point.

Claim. There exists a constant $C_{0}$ depending on $f$ such that

$$
m \leq C_{0}+2 \ell_{\max } \log (1 / \varepsilon) / \log r_{1},
$$

where $r_{1}=r / 4^{\ell_{\max }}$.

In fact, $\widehat{W}_{n_{1}} \subset \widetilde{B}\left(\delta_{0}\right)$. By the $\mathrm{BC}(r)$ property, we deduce that $\widehat{W}_{n_{2}}^{\prime} \subset$ $\widetilde{B}\left(\delta_{0} / r\right)$, so that $\widehat{W}_{n_{2}} \subset \widetilde{B}\left(4^{\ell_{\max }} \delta_{0} / r\right) \subset \widetilde{B}\left(\delta_{0}\right)$. Repeating the procedure, we obtain

$$
\widehat{W}_{n_{m}} \subset \widetilde{B}\left(\delta_{0} / r_{1}^{m-1}\right) .
$$

By Proposition 2 , for any $\beta \in\left(0,1 / \ell_{\max }\right)$, there exists $C(\beta)>0$ such that

$$
\operatorname{diam}(W) \leq C(\beta)\left(\delta_{0} / r_{1}^{m-1}\right)^{\beta} .
$$

Since $\operatorname{diam}(W) \geq \operatorname{IR}(W)=\varepsilon^{1+\alpha / 2 t} \geq \varepsilon^{1+\alpha}$, we obtain (5).

For each $1 \leq i \leq m, f^{n_{i-1}-n_{i}-1}: W_{n_{i}+1} \rightarrow W_{n_{i-1}}$ extends to a conformal map onto $\widehat{W}_{n_{i-1}}$. Since $\bmod \left(\widehat{W}_{n_{i-1}} \backslash W_{n_{i-1}}\right)$ is bounded away from zero, the Koebe principle and the $t$-covariance of $\mu$ give us

$$
\begin{aligned}
\frac{\mu\left(W_{n_{i}+1}\right)}{\operatorname{diam}\left(W_{n_{i}+1}\right)^{t}} & \geq C_{1} \frac{\mu\left(W_{n_{i-1}}\right)}{\operatorname{diam}\left(W_{n_{i-1}}\right)^{t}}, \\
\frac{\operatorname{OR}\left(W_{n_{i}+1}, f^{n_{i}+1}(z)\right)}{\operatorname{IR}\left(W_{n_{i}+1}, f^{n_{i}+1}(z)\right)} & \leq \frac{1}{C_{1}} \frac{\operatorname{OR}\left(W_{n_{i-1}}, f^{n_{i-1}}(z)\right)}{\operatorname{IR}\left(W_{n_{i-1}}, f^{n_{i-1}}(z)\right)},
\end{aligned}
$$

where $C_{1} \in(0,1)$ is a universal constant. Similarly, we have

$$
\begin{aligned}
& \frac{\mu(W)}{\operatorname{diam}(W)^{t}} \geq C_{1} \frac{\mu\left(W_{n_{m}}\right)}{\operatorname{diam}\left(W_{n_{m}}\right)^{t}}, \\
& \frac{\operatorname{OR}(W, z)}{\operatorname{IR}(W, z)} \leq \frac{1}{C_{1}} \frac{\operatorname{OR}\left(W_{n_{m}}, f^{n_{m}}(z)\right)}{\operatorname{IR}\left(W_{n_{m}}, f^{n_{m}}(z)\right)} .
\end{aligned}
$$

By Lemma 5, we have

$$
\frac{\mu\left(W_{n_{i}}\right)}{\operatorname{diam}\left(W_{n_{i}}\right)^{t}} \geq C_{2} \frac{\mu\left(W_{n_{i}+1}\right)}{\operatorname{diam}\left(W_{n_{i}+1}\right)^{t}}
$$


where $C_{2} \in(0,1)$ is a universal constant. By Proposition 4, we have

$$
\frac{\mathrm{OR}\left(W_{n_{i}}, f^{n_{i}}(z)\right)}{\operatorname{IR}\left(W_{n_{i}}, f^{n_{i}}(z)\right)} \leq \frac{1}{C_{2}} \frac{\mathrm{OR}\left(W_{n_{i}+1}, f^{n_{i}+1}(z)\right)}{\operatorname{IR}\left(W_{n_{i}+1}, f^{n_{i}+1}(z)\right)} .
$$

Combining the estimates (6), (10) and (8), we obtain

$$
\frac{\mu(W)}{\operatorname{diam}(W)^{t}} \geq C_{1}^{m+1} C_{2}^{m} \frac{\mu\left(B\left(f^{n}(z), \eta^{\prime}\right)\right)}{\left(2 \eta^{\prime}\right)^{t}} .
$$

Since $\inf _{w \in J(f)} \mu\left(B\left(w, \eta^{\prime}\right)\right)>0$, it follows that

$$
\frac{\mu(W)}{\operatorname{diam}(W)^{t}} \geq C\left(C_{1} C_{2}\right)^{m}
$$

where $C$ is a constant.

Combining the estimates (7), (9) and (11), we obtain

$$
\frac{\mathrm{OR}(W, z)}{\operatorname{IR}(W, z)} \leq\left(C_{1}^{m+1} C_{2}^{m}\right)^{-1}
$$

If we let $\theta=-\log \left(C_{1} C_{2}\right)$ and redefine the constant $C$, then (13) and (14) give us (3) and (4) respectively. This completes the proof.

\section{Proof of the Main Theorem}

Proof of the Main Theorem in the case $J(f) \neq \overline{\mathbb{C}}$. The following argument is similar to the proof of the well-known Frostman's lemma (see [8]). Since $\delta_{*}(f)=\operatorname{HD}_{\text {hyp }}(J(f))$, it suffices to prove that $\overline{\mathrm{BD}}(J(f)) \leq \delta_{*}(f)+\alpha$ for any $\alpha>0$.

Let $\mu$ be a $\delta_{*}(f)$-conformal measure of $f$. By Lemma $3, f$ satisfies the assumption $(*)$, so that we can apply Theorem 1 . Let $N(\varepsilon)$ be the minimal number of open balls with radius $\varepsilon$ needed to cover $J(f)$. For any $\varepsilon>0$ small, $J(f)$ can be covered by a family $\left\{B_{i}\right\}_{i=1}^{n}$ of open balls of radius $\varepsilon$ with intersection multiplicity 4 . For each $i$, Theorem 1 gives us $\mu\left(B_{i}\right) \geq \varepsilon^{\delta_{*}(f)+\alpha}$, provided that $\varepsilon$ is small enough. Thus

$$
4 \geq \sum_{i=1}^{n} \mu\left(B_{i}\right) \geq n \varepsilon^{\delta_{*}(f)+\alpha} \geq N(\varepsilon) \varepsilon^{\delta_{*}(f)+\alpha},
$$

which implies that

$$
\overline{\mathrm{BD}}(J(f))=\limsup _{\varepsilon \rightarrow 0} \frac{\log N(\varepsilon)}{\log (1 / \varepsilon)} \leq \delta_{*}(f)+\alpha .
$$

In the case $J(f)=\overline{\mathbb{C}}$, we do not know whether the condition $(*)$ holds. However, we are still able to obtain enough control on the conformal measures to conclude the proof.

Proposition 5. If $J(f)=\overline{\mathbb{C}}$, then $\delta_{*}(f)=2$. 
Proof. Arguing by contradiction, assume that $f$ has a $t$-conformal measure with $t<2$.

Let $r>1$ be a large constant. Since $f$ has the $\mathrm{BC}(r)$ property, there exists an arbitrarily small $\varepsilon>0$ and a puzzle neighborhood $V=\bigcup_{c \in \operatorname{Crit}^{\prime}(f)} V_{c}$ of $\operatorname{Crit}^{\prime}(f)$ such that for each $c \in \operatorname{Crit}^{\prime}(f)$,

$$
\widetilde{B}(c, \varepsilon) \subset V_{c} \subset \widetilde{B}(c, 2 \varepsilon) .
$$

Let $W_{c}$ be the union of all return domains of $V$ which are not contained in $\widetilde{B}(c, \varepsilon / \sqrt{r})$. Let $g: \bigcup_{c} W_{c} \rightarrow V$ denote the first return map into $V$ under iteration of $f$. By the $\mathrm{BC}(r)$ property, for each component $U$ of $g^{-n}(V)$, $g^{n}: U \rightarrow V$ is a conformal map which extends to a conformal map onto $\widetilde{B}(c, \sqrt{r} \varepsilon)$ for some $c \in \operatorname{Crit}^{\prime}(f)$. In fact, this follows from the following observation: if $U$ is a component of $W_{c}$ with $g(U)=V_{c^{\prime}}$ and $g\left|U=f^{s}\right| U$, then there exists a topological disk $\widehat{U} \subset \widetilde{B}(c, \sqrt{r} \varepsilon)$ such that $f^{s}: \widehat{U} \rightarrow$ $\widetilde{B}\left(c^{\prime}, \sqrt{r} \varepsilon\right)$. By the Koebe principle, it follows that for each component $U$ of $g^{-n}(V), g^{n} \mid U$ has small distortion provided that $r$ is large enough.

Let $\mathcal{W}_{c}^{n}$ be the collection of all components of $g^{-n}(V)$ which are contained in $V_{c}$ and let $W_{c}^{n}$ be the union of these components. By Lemma 1, almost every point returns to $V$ under iteration of $f$, thus,

$$
\frac{\operatorname{area}\left(W_{c}^{1}\right)}{\operatorname{area}\left(V_{c}\right)} \geq 1-\frac{\widetilde{B}(c, \varepsilon / \sqrt{r})}{\widetilde{B}(c, \varepsilon)} \geq 1-\sigma(r),
$$

where $\sigma(r) \rightarrow 0$ as $r \rightarrow \infty$. Note that for each component $U$ of $W_{c}^{1}$, $\operatorname{area}(U) \leq \operatorname{area}\left(V_{c}\right) / 2$. Therefore, provided that $r$ was chosen large enough, we have

$$
\sum_{U \in \mathcal{W}_{c}^{1}} \operatorname{area}(U)^{t / 2} \geq \lambda \operatorname{area}\left(V_{c}\right)^{t / 2}
$$

where $\lambda=2^{1-t / 2}>1$.

For each $U \in \mathcal{W}_{c}^{n}$, since $g^{n} \mid U$ has small distortion and $g^{n}$ maps an element of $\mathcal{W}_{c}^{n+1}$ onto an element of $\mathcal{W}_{c}^{1}$, it follows that

$$
\sum_{W \in \mathcal{W}_{c}^{n+1}, W \subset U} \operatorname{area}(W)^{t / 2} \geq \lambda_{1} \operatorname{area}(U)^{t / 2},
$$

where $\lambda_{1} \in(1, \lambda)$. Therefore,

$$
\sum_{U \in \mathcal{W}_{c}^{n}} \operatorname{area}(U)^{t / 2} \geq \lambda_{1}^{n} \operatorname{area}\left(V_{c}\right)^{t / 2}
$$

By the Koebe principle and the $t$-covariance of $\mu$, for each $U \in \mathcal{W}_{c}^{n}, n=$ $0,1, \ldots$, we know that $\mu(U) / \operatorname{area}(U)^{t / 2}$ is comparable to $\mu\left(V_{c}\right) / \operatorname{area}\left(V_{c}\right)^{t / 2}$, 
where $V_{c}=g^{n}(U)$. Thus,

$$
\sum_{U \in \mathcal{W}_{c}^{n}} \mu(U) \geq C \lambda_{1}^{n} .
$$

Letting $n \rightarrow \infty$ implies $\mu(J(f))=\infty$, a contradiction.

Proof of the Main Theorem in the case $J(f)=\overline{\mathbb{C}}$. By the previous proposition, $\delta_{*}(f)=2$. Hence

$$
\delta_{*}(f)=\mathrm{HD}_{\text {hyp }}(f)=2=\overline{\mathrm{BD}}(J(f)) .
$$

\section{References}

[1] H. Bruin, J. Rivera-Letelier, W. X. Shen and S. van Strien. Large derivatives, backward contraction and invariant densities for interval maps, preprint, 2007.

[2] M. Denker and M. Urbański, On Sullivan's conformal measures for rational maps of the Riemann sphere, Nonlinearity 4 (1991), 365-384.

[3] K. Falconer, Fractal Geometry. Mathematical Foundations and Applications, Wiley, 1990.

[4] J. Graczyk and S. Smirnov, Non-uniform hyperbolicity in complex dynamics, I, II, Invent. Math., to appear.

[5] J. Milnor, Dynamics in One Complex Variable: Introductory Lectures, Vieweg, Braunschweig, 1999.

[6] F. Przytycki, Iterations of holomorphic Collet-Eckmann maps: conformal and invariant measures, Trans. Amer. Math. Soc. 350 (1998), 717-742.

[7] F. Przytycki and J. Rivera-Letelier, Statistical properties of topological Collet-Eckmann maps, Ann. Sci. École Norm. Sup. 40 (2007), 135-178.

[8] F. Przytycki and M. Urbański, Fractals in the Plane-the Ergodic Theory Methods, http://www.math.unt.edu/ urbanski/.

[9] J. Rivera-Letelier, A connecting lemma for rational maps satisfying a no growth condition, Ergodic Theory Dynam. Systems 27 (2007), 595-636.

[10] D. Sullivan, Conformal dynamical system, in: Geometric Dynamics, Lecture Notes in Math. 1007, Springer, 1983, 725-752.

[11] M. Urbański, Measures and dimensions in conformal dynamics, Bull. Amer. Math. Soc. 40 (2003), 281-321.

Mathematics Department

University of Science and Technology of China

Hefei 230026, China

E-mail: hbli@mail.ustc.edu.cn

wxshen@ustc.edu.cn

Received 6 October 2007;

in revised form 29 October 2007 